\title{
Psychosocial functioning in pediatric heart transplant recipients and their families
}

\author{
Melissa K. Cousino ${ }^{1,2}$ (i) | Kurt R. Schumacher ${ }^{1,2}$ | Kelly E. Rea ${ }^{1}$ | Sally Eder ${ }^{1}$ | Mary \\ Zamberlan $^{3}$ | Jessica Jordan ${ }^{1}$ | Emily M. Fredericks ${ }^{1,2,4}$
}

${ }^{1}$ Department of Pediatrics, Michigan

Medicine, Ann Arbor, MI, USA

${ }^{2}$ University of Michigan Transplant Center, Ann Arbor, MI, USA

${ }^{3}$ Department of Cardiac Surgery, Michigan Medicine, Ann Arbor, MI, USA

${ }^{4}$ Child Health Evaluation and Research Center, University of Michigan, Ann Arbor, MI, USA

\section{Correspondence}

Melissa K. Cousino, Department of Pediatrics, Michigan Medicine, Ann Arbor, MI, USA.

Email: melcousi@med.umich.edu

Funding information

University of Michigan Transplant Center

\begin{abstract}
Across pediatric organ transplant populations, patient and family psychosocial functioning is associated with important health-related outcomes. Research has suggested that pediatric heart transplant recipients and their families are at increased risk for adverse psychosocial outcomes; however, recent investigation of psychosocial functioning in this population is lacking. This study aimed to provide a contemporary characterization of psychosocial functioning in pediatric heart transplant recipients and their families. Associations between psychosocial function, demographic variables, and transplant-related variables were investigated. Fifty-six parents/guardians of pediatric heart transplant recipients completed a comprehensive psychosocial screening measure during transplant follow-up clinic visits. Descriptive statistics, correlational analyses, and independent samples $t$ tests were performed. Forty percent of pediatric heart transplant recipients and their families endorsed clinically meaningful levels of total psychosocial risk. One-third of patients presented with clinically significant psychological problems per parent report. Psychosocial risk was unassociated with demographic or transplant-related factors. Despite notable improvements in the survival of pediatric heart transplant recipients over the past decade, patients and families present with sustained psychosocial risks well beyond the immediate posttransplant period, necessitating mental health intervention to mitigate adverse impact on health-related outcomes.
\end{abstract}

\section{KEYWORDS}

heart transplant, pediatric transplant, psychosocial functioning, psychosocial screening

\section{1 | INTRODUCTION}

As a result of continued research and advancements in clinical practice, 5-year survival rates for pediatric heart transplant recipients are approaching or exceeding $80 \%$, depending on age at transplant. ${ }^{1}$ Given that the purpose of transplantation is not only to extend life but also to enhance the quality of life among this population, ${ }^{2}$ attention to outcomes other than survival is needed. With increasing longevity of pediatric heart transplant recipients, it is important to evaluate

Abbreviations: PAT, Psychosocial Assessment Tool; PPPHM, Pediatric Psychosocial Preventative Health Model; SD, standard deviations. the emotional, behavioral, and social functioning of these patients and their families throughout their post-transplant life. Furthermore, underscoring its importance, recipient and parent psychosocial functioning is associated with health-related outcomes in pediatric solid organ transplant populations, including adherence to the immunosuppressant regimen ${ }^{3,4}$ and number of hospitalizations. ${ }^{5}$

Results of limited studies have suggested that while the majority of pediatric heart transplant recipients report normative psychological health, a subset of recipients are at increased risk for long-term sequelae. Approximately $30 \%$ of pediatric heart transplant recipients have been found to have clinically significant mental health problems 
post-transplant. ${ }^{2,6,7}$ Parent and family functioning has also been found to be impaired in a notable portion of pediatric heart transplant recipient families, ${ }^{8,9}$ which in turn is associated with more adverse psychological outcomes for patients themselves. ${ }^{6,10}$

While these early studies underscore the value of assessing psychosocial functioning in pediatric heart transplant recipients and their families given its potential impact on health outcomes, the majority of published work is now quite dated and evaluated small patient samples. A recent systematic review of family functioning in pediatric solid organ transplant populations identified only five studies, all of which were greater than a decade old, that assessed parent psychological and/or family functioning in pediatric heart transplant samples. ${ }^{11}$ To our knowledge, no studies to date have screened for sibling emotional and behavioral problems in pediatric heart transplant populations. With improvements in pediatric heart transplant outcomes and advancements in post-transplant care over the past decade, it is possible that the psychosocial impact of pediatric heart transplantation on patients and families has also changed. Thus, a contemporary and expanded characterization of psychosocial functioning in pediatric heart transplant recipients and their families is needed to guide current practice and research.

This study aimed to provide an updated assessment of psychosocial functioning in pediatric heart transplant recipients, siblings, and parent/caregivers through the use of a validated global screening tool. A secondary aim was to evaluate use of this screening tool, the PAT, ${ }^{12}$ in the pediatric heart transplant population. The PAT, which is freely available, has been used in pediatric cancer survivor, ${ }^{13}$ infant congenital heart, ${ }^{14}$ and kidney and liver transplant populations. ${ }^{15,16}$ It was selected for this study due to its brief, yet comprehensive assessment of various domains of both modifiable and non-modifiable psychosocial risks, including family resources and support, patient and sibling emotional and behavioral functioning, and parent/family stress, mental health, and illness-related beliefs. Thirdly, associations between psychosocial functioning, patient demographic variables, and transplant-related variables were also investigated. Per previous literature, ${ }^{11,17}$ it was hypothesized that psychosocial risk would be equally distributed across the sample, with no associations between demographic variables, primary disease, and time since transplant.

\section{2 | PATIENTS AND METHODS}

\section{1 | Participants and methods}

The current investigation was approved as a non-regulated study by the institutional review board at the University of Michigan Medical School. Legal guardians of pediatric transplant recipients of any age and time since transplant were asked to participate in this newly launched annual psychosocial screening program. Parents or legal guardians of 56 pediatric heart transplant recipients (ages 0-22 years) completed the psychosocial screening measure during their child's regularly scheduled transplant follow-up clinic visit. The paper-pencil screening measure was given to parent/guardian by the clinic's medical assistant upon being roomed. Time to complete was generally 10 minutes or less, and parents/guardians completed the screening measure while waiting to see the various providers. Upon completion, the screening measure was given to the pediatric transplant psychologist or psychology trainee who briefly reviewed items prior to meeting with patient and families. The screening measure was used to guide in-person assessment and discussion. Relevant results were also shared with clinic social worker prior to their meeting with patient and family. The screening measure was not regularly scored prior to mental health clinician seeing the patient due to high patient volume and needs during a busy clinic. Rather, it was scored following clinic and used to ensure patients and families with clinically significant psychosocial risks were scheduled for follow-up visits with dedicated time to meet with psychology and/or social work. Among our active post-transplant patient census, $84 \%$ of patients' parents/caregivers completed the screening measure. Common reasons for not completing the psychosocial screening measure included primary language other than English or Spanish $(\mathrm{N}=1)$, parent not present at appointment with patient 18 years of age or older $(\mathrm{N}=3)$, psychology clinician unable to meet with patient/family for screening ( $N=6)$, or parent refused to complete $(N=1)$.

\subsection{Measures}

Given its established use in other pediatric solid organ transplant populations, ${ }^{15,16}$ parents/caregivers completed the psychometrically sound, validated PAT as a broad-based assessment of psychosocial risk. ${ }^{12,18}$ The PAT includes seven subscales: family structure/ resources (eg, finances and number of children), family social support, child emotional/behavioral problems (eg, mood, anxiety, development/learning, sleep, attention/behavior, and social functioning), sibling emotional/behavioral problems, family problems (eg, parent depression, anxiety, and marital discord), parent stress reactions (eg, intrusive memories/thoughts of child being sick, avoidance of places, people or things that remind the parent of their child being sick, hypervigilance to symptoms), and family beliefs (eg, beliefs related to child's illness or health system). PAT scores are conceptualized into a pyramid based on the PPPHM, used to describe the degree of patient and familial risk and determine the level of intervention needed. Based upon the PPPHM pyramid (see Figure 1), the majority of families fall into the bottom risk category-reporting some degree of stress, but also a number of protective factors (Universal Risk). In the middle are families with moderate risks requiring targeted interventions (Targeted Risk). At the top of the pyramid are those who indicate high levels of distress and multiple psychosocial risk factors (Clinical Risk). Those within the Clinical Risk range necessitate a high degree of psychological and supportive interventions. To determine risk range, a total score ranging from 0 to 7 is calculated. Total scores of $<1$ indicate Universal Risk, 1 to $<2$ indicate Targeted Risk, and $\geq 2$ indicate Clinical Risk. Subscale scores of $\geq 0.5$ are clinically significant. ${ }^{12,18}$ Patient and family demographic variables were obtained via the PAT. Patient diagnosis, age at transplant, and time since transplant were obtained via medical chart review. 
FIGURE 1 Pediatric Psychosocial Preventative Health Model

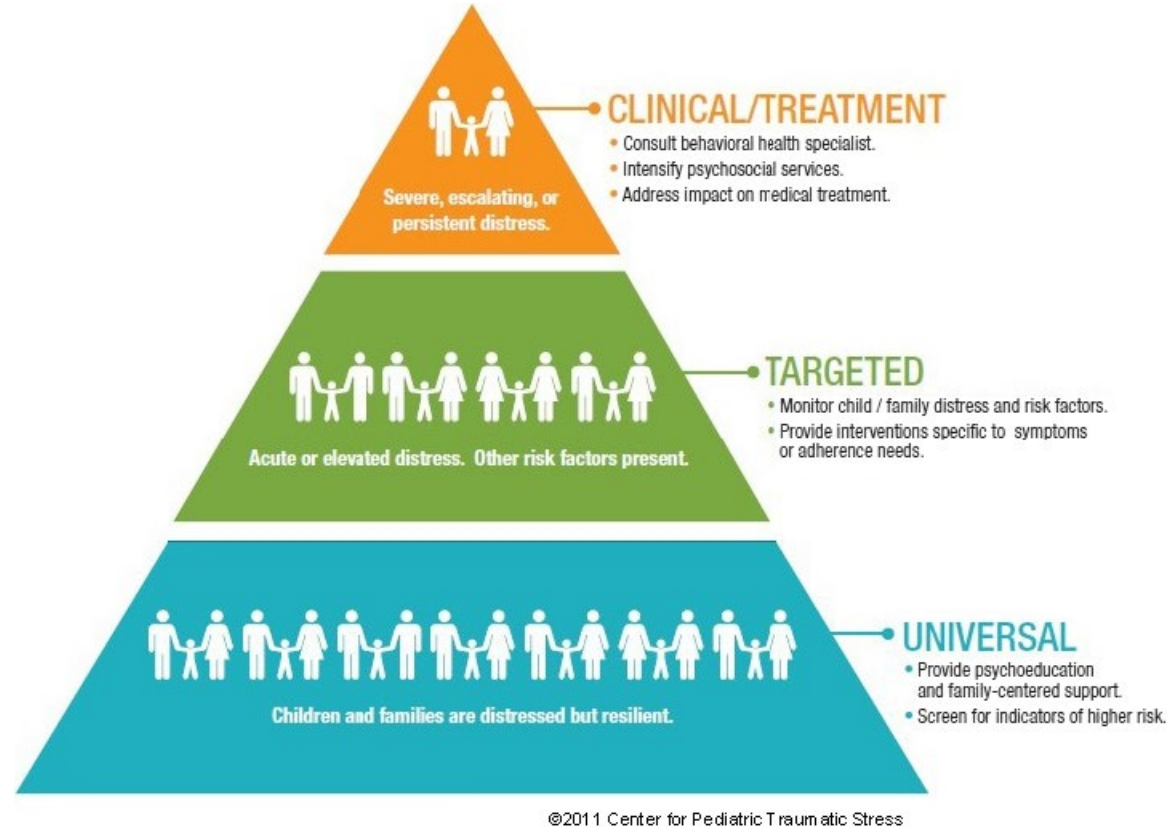

TABLE 1 Patient demographics

\subsection{Statistical analysis}

Preliminary analyses were conducted including descriptive analyses for the PAT, PAT total score, and subscale internal consistencies, as well as correlational and intergroup analyses to assess the association between demographic factors and PAT scores. Descriptive statistics are presented as frequencies and percentages for categorical variables, and means and SD for continuous variables. All analyses were performed using a statistical significance level of 0.05 using two-sided tests. Pearson correlation coefficients were calculated to detect associations between psychosocial risk, demographic, and health-related variables. Independent samples $t$ test was utilized to test differences in psychosocial risk based upon primary diagnosis (congenital heart disease, $\mathrm{N}=21$ vs cardiomyopathy/acquired heart disease, $\mathrm{N}=34$ ). Post hoc power analysis demonstrated ability to detect disease group differences at 0.70 level. The PAT total score was used as the comprehensive measure of psychosocial risk in correlational and $t$ test analyses.

\section{3 | RESULTS}

\subsection{Sample demographics and descriptive statistics}

Patient demographic and health-related data are reported in Table 1. A total of 56 parents/legal guardians, predominately mothers (79\%), completed the PAT. Parent respondent marital status was as follows: married/partnered (54\%), single (28\%), and separated/divorced (17\%). Of the 56 responding parents, 28 completed college/trade school and five completed a masters or doctoral program. Five parents indicated that they did not complete high school. Per parent report, the majority of patients were insured via Medicaid (61\%). Descriptive statistics and internal consistencies of the PAT total and subscale scores are provided in Table 2.

\begin{tabular}{|c|c|}
\hline Characteristic & $n=56$ \\
\hline Mean patient age in years (SD) & $11.90(5.94)$ \\
\hline Mean patient age at transplant in years (SD) & $6.00(5.85)$ \\
\hline Mean years since transplant (SD) & $5.93(4.89)$ \\
\hline \multicolumn{2}{|l|}{ Patient sex (\%) } \\
\hline Female & $24(42.9)$ \\
\hline Male & $32(57.1)$ \\
\hline \multicolumn{2}{|l|}{ Patient race/ethnicity (\%) } \\
\hline White/Caucasian & $40(71.4)$ \\
\hline Black/African American & $11(19.6)$ \\
\hline $\mathrm{Bi} /$ multiracial & $3(5.4)$ \\
\hline Other & $2(3.6)$ \\
\hline \multicolumn{2}{|l|}{ Patient diagnosis ${ }^{a}(\%)$} \\
\hline Single ventricle & $21(38.2)$ \\
\hline Dilated cardiomyopathy & $15(27.3)$ \\
\hline Restricted cardiomyopathy & $6(10.9)$ \\
\hline Other cardiomyopathy & $6(10.9)$ \\
\hline Myocarditis & $2(3.6)$ \\
\hline Other & $5(9.1)$ \\
\hline \multicolumn{2}{|l|}{ Parent/caregiver respondent ${ }^{\mathrm{b}}(\%)$} \\
\hline Mother & $41(78.8)$ \\
\hline Father & $10(19.2)$ \\
\hline Other & $1(2.0)$ \\
\hline
\end{tabular}

${ }^{a}$ One family did not respond. $\mathrm{n}=55$.

${ }^{b}$ Four families did not respond. $\mathrm{n}=52$.

\subsection{Patient and family psychosocial risk}

Psychosocial risk distribution among the sample is detailed in Table 3. The majority of pediatric heart transplant recipients and their families 
TABLE 2 Psychosocial assessment tool descriptive statistics and internal consistencies

\begin{tabular}{|c|c|c|c|c|}
\hline Scale & Mean & SD & Range & $\begin{array}{l}\text { Reliability } \\
\text { coefficient }\end{array}$ \\
\hline $\begin{array}{l}\text { Family } \\
\text { structure/ } \\
\text { resources }\end{array}$ & 0.15 & 0.17 & $0-0.71$ & 0.48 \\
\hline $\begin{array}{l}\text { Family social } \\
\text { support }\end{array}$ & 0.08 & 0.21 & $0-1.00$ & 0.77 \\
\hline $\begin{array}{l}\text { Child (patient) } \\
\text { problems }\end{array}$ & 0.31 & 0.23 & $0-0.89$ & 0.83 \\
\hline $\begin{array}{l}\text { Sibling } \\
\text { problems }\end{array}$ & 0.28 & 0.20 & $0-0.45$ & 0.86 \\
\hline Family beliefs & 0.09 & 0.10 & $0-0.58$ & 0.59 \\
\hline $\begin{array}{l}\text { Family } \\
\text { problems }\end{array}$ & 0.15 & 0.14 & $0-0.50$ & 0.45 \\
\hline $\begin{array}{l}\text { Parent stress } \\
\text { reactions }\end{array}$ & 0.13 & 0.25 & $0-1.00$ & 0.81 \\
\hline Total score & 0.96 & 0.75 & $0-2.98$ & 0.89 \\
\hline
\end{tabular}

fell within the Universal Risk category (59\%). These parents/guardians reported low levels of child, sibling, parent, and/or family problems. However, a significant portion of the sample fell within risk categories indicating need for psychosocial intervention (41\%). Specifically, 30\% of the sample endorsed stressors and problems within the Targeted Risk category and $11 \%$ fell within the Clinical Risk category.

With regard to subscale scores, $30 \%$ of parents/legal guardians endorsed clinically significant patient-specific problems. No parents/ legal guardians reported clinically significant sibling issues. Although very few parents/legal guardians endorsed clinically significant family problems (2\%), such as parental depression, anxiety, substance use, or relational problems, $9 \%$ of respondents reported high levels of caregiver stress reactions. Overall, the majority of parents reported having appropriate resources (eg, financial and transportation) and caregiving supports available.

Among the transplant recipients with clinically significant patientspecific problems, 53\% were referred by transplant psychology for additional outpatient psychology and/or neurodevelopmental evaluation and treatment. The majority of these patients and families followed through with referral. One patient was currently in local counseling, and continued local treatment was encouraged. The remaining patients with clinically significant patient problems (41\%) were flagged for psychology assessment with continued monitoring of psychosocial functioning at their next transplant clinic visit. Among this group not referred for additional psychological evaluation and treatment at time of screening, most presented with learning/developmental risks and previous neurodevelopmental screening had been completed or early-on/therapy services were already in place.

No patient demographic, temporal, or historical medical factors were associated with the PAT total score. Patient age at time of psychosocial screening $(r=-.03, P=.80)$, sex $(r=-.16, P=.25)$, and race $(r=-.20, P=.13)$ were not associated with psychosocial risk. Similarly, age at transplant $(r=.05, P=.73)$ and time since transplant were
TABLE 3 Patient and family psychosocial risk distribution

\begin{tabular}{|lr|}
\hline PAT scale & $\mathbf{n}=56$ \\
\hline Total risk (\%) & $6(10.7)$ \\
\hline Clinical & $17(30.4)$ \\
\hline Targeted & $33(58.9)$ \\
\hline Universal & \\
\hline Child (patient) problems (\%) & $17(30.4)$ \\
\hline Clinical & $39(69.6)$ \\
\hline Non-clinical & \\
\hline Sibling problems (\%) & $0(0.0)$ \\
\hline Clinical & $56(100)$ \\
\hline Non-clinical & \\
\hline Parent stress reactions (\%) & $5(9.1)$ \\
\hline Clinical & $50(90.9)$ \\
\hline Non-clinical
\end{tabular}

unrelated to psychosocial risk ( $r=-.12, P=.38$ ). Independent samples $t$ test revealed no significant differences in overall psychosocial risk among those with congenital heart disease $(M=0.82, S D=0.50)$ and those with a cardiomyopathy or other acquired heart disease $(M=1.0$, $\mathrm{SD}=0.84 ; t(53)=-0.93, P=.36)$. Similarly, there were no significant differences in patient psychosocial risk among the congenital heart disease $(M=0.28, S D=0.20)$ and cardiomyopathy/other acquired heart disease groups $(M=0.33, S D=0.25$.; $t(49)=-0.76, P=.45)$.

\section{4 | DISCUSSION}

\section{1 | Patient and family psychosocial risk}

While pediatric heart transplantation offers increased survival and improved physical functioning for many children with end-stage heart failure, solid organ transplantation is a chronic condition, with long-term risk for rejection, comorbid medical conditions (eg, kidney disease, diabetes, post-transplant lymphoma), and eventual graft loss with potential need for retransplantation. Treatment demands are notable post-heart transplant, with frequent need for biopsies, cardiac catheterizations, and daily medications. ${ }^{19}$ Results of the current study demonstrated that approximately $40 \%$ of pediatric heart transplant recipients and their families endorsed clinically meaningful levels of overall psychosocial risk, continuing well beyond the immediate post-transplant period. Consistent with previous psychosocial research, ${ }^{6,7,20}$ parent respondents described clinically significant child emotional and behavioral problems in $30 \%$ of the recipient sample. Although sibling emotional and behavioral problems were assessed with the PAT, parents did not indicate any concerns specific to sibling functioning. At the parent level, $9 \%$ of study respondents endorsed clinically significant levels of caregiver stress reactions.

When compared to other illness groups, rates of psychosocial risk in heart transplant recipients and their families are similar to those reported by parents of children newly diagnosed with cancer ${ }^{21,22}$ and 
children 2 years post-cancer treatment. ${ }^{13}$ Rates of those falling within the Clinical Risk range among the current sample were approximately 5 times those of the infant CHD sample. ${ }^{14}$ This may be due to the increased presence of patient-specific problems (eg, depression and behavioral issues) in the current study's older transplant population, as well as greater time living with a chronic, life-threatening disease.

\section{2 | Psychosocial risk, and demographic and health- related factors}

Patient and family psychosocial distress can negatively impact important recipient-related outcomes, such as adherence to immunosuppressant regimes. ${ }^{4,23}$ As a result, adverse psychosocial outcomes can be incredibly costly for pediatric heart transplant recipientspotentially resulting in rejection, graft loss, or death in the most extreme of cases. In an effort to inform clinical practice and identify those at greatest risk for such negative outcomes, this study also examined associations between psychosocial risk, demographic variables, and transplant-related factors. Demographic variables, including child age, sex, and race, were unrelated to overall psychosocial distress. Findings are generally consistent with the literature, where null associations between parent and family functioning, child age, and child sex have been reported. ${ }^{11}$ Similarly, age at transplant and time since transplant were not associated with psychosocial risk, which is consistent with findings among pediatric liver transplant recipients. ${ }^{24}$ There were no differences in psychosocial risk among recipients with congenital heart diseases when compared to those with a cardiomyopathy or other acquired heart disease. As a result of these null associations, global psychosocial screening of all recipients and their families is recommended. As the current study demonstrates, such screening is feasible in a transplant clinic setting and helps to identify those in need of more intensive psychosocial interventions until predictive factors are better understood through future research.

\section{5 | CLINICAL IMPLICATIONS}

Overall, despite notable improvements in both the survival and the medical management of pediatric heart transplant recipients over the past decade, rates of patient and family psychosocial problems remain largely the same. Although rates of psychosocial risk in pediatric heart transplant recipients are similar to those found in pediatric oncology populations, ${ }^{13,21,22}$ psychosocial research and integrated clinical care in pediatric heart transplantation lags behind when compared to pediatric cancer. For example, Pediatric Blood \& Cancer recently featured 17 articles comprising the Standards for Psychosocial Care for Children with Cancer and their Families. ${ }^{25}$ These clinical guidelines have been adopted by various national and international cancer organizations and medical societies, and psychosocial providers are commonly involved as integrated members of multidisciplinary pediatric cancer care teams. While pediatric-specific standards for psychosocial care in heart transplant do not exist, the 2010 International
Society for Heart and Lung Transplantation Guidelines for the care of heart transplant recipients ${ }^{26}$ recommends that each heart transplant center collaborate with or include a team psychologist for regular assessment and treatment of non-adherence and depression. Per informal e-survey of Pediatric Heart Transplant Study membership in 2015 , fewer than $40 \%$ of responding pediatric heart transplant programs had access to psychology/psychiatry consult services and only 1 in 5 programs reported having integrated psychology/psychiatry providers as part of their team.

With rates of clinically significant psychosocial problems exceeding $40 \%$ among this population, psychosocial providers, including psychologists and social workers, should be integrated members of the transplant team-providing psychosocial assessment, referral, and intervention in conjunction with medical follow-up visits. It is important that screening and intervention continue well beyond the transplant evaluation period given the persisting psychosocial distress reported by parents. Due to limited time, quick and easy-toadminister screening measures, such as the PAT, can be utilized to identify patients and families in greatest need of intervention. Those reporting high levels of risk can be provided in-clinic interventions, such as psychoeducation about a variety of issues (eg, sleep hygiene, behavioral management, school services, and relaxation strategies for anxiety), clinician-supported problem-solving specific to family stressors, or referral for more intensive patient and/or parent psychological evaluation and treatment. It is also important that clinical care and research move beyond the identification of problems through the development and evaluation of transplant-specific clinical interventions. While adherence-specific patient interventions have been reported on, ${ }^{27,28}$ there have been no family-based intervention studies aimed at improving family functioning and decreasing parental stress in transplant populations. Single-day family-based cognitive behavioral interventions that have successfully reduced anxiety and post-traumatic stress symptoms in childhood cancer populations and their families ${ }^{29}$ can be adapted and evaluated among pediatric transplant populations.

\section{I LIMITATIONS AND FUTURE RESEARCH DIRECTIONS}

It is important to highlight that results must be considered in light of study limitations. First, as is the case with many pediatric transplant studies, the single center sample size is small. While moderately powered, this may have limited the ability to detect associations between psychosocial risk, demographic, and transplant-related factors. Secondly, although the PAT is a quick and easy-to-administer psychosocial screening measure, it is based upon parent report only. Research has shown that parents and youth often have discordant reports of child psychological functioning. ${ }^{30}$ Similarly, underreporting among the entire sample may be present. Some families reported no issues on the PAT measure, but endorsed a number of problem areas during clinical interview and review of the screening measure with the program psychologist. This would mean that our sample 
underestimates that degree of psychosocial impairment, making assessment even more necessary. Lastly, the PAT was administered at a single clinic visit over the course of 1 year. For some pediatric heart transplant recipients and their families, news of rejection or need for hospitalization presented following completion of the PAT. Thus, the annual screening measure did not always capture the changing and unpredictable stressors that may present throughout the posttransplant course.

In light of such limitations, future research should seek to recruit larger samples of heart transplant recipients across multiple transplant centers. National and international pediatric transplant databases should consider the addition of psychosocial measures to enhance our understanding of this notable problem among a population in need of increased psychological intervention. Brief psychosocial screening measures aide providers in quickly identifying patient and family risks and needs that necessitate interventions; however, there is a notable lack of valid, reliable, and quick-toadminister comprehensive screening measures. Although the PAT covers various domains of family and patient functioning, it is based upon parent report only. The development of an adolescent/young adult version of this measure could be a noteworthy undertaking for future research. Additionally, longitudinal assessment of psychosocial functioning will hopefully aid in the identification of time points or events leading to increased risk and distress for patients and families. Other health variables, such as treatment adherence, number of hospitalizations, rejection episodes, and transplantrelated complications, as well as additional psychosocial correlates, including disease self-efficacy, fear of complications/retransplantation, and disease knowledge, are important factors to consider in future research in order to identify precipitating factors of increased psychosocial risk post-transplant. With over $40 \%$ of pediatric heart transplant recipients and their families experiencing clinically significant psychosocial stressors, action is necessary to improve both physical and mental health outcomes.

\section{ACKNOWLEDGMENTS}

This work was supported by funding provided by the University of Michigan Transplant Center. The authors have no financial disclosures or industry relationships pertinent to this work to report.

\section{AUTHORS' CONTRIBUTION}

Melissa K. Cousino: Involved in concept/design, data collection, data analysis/interpretation, statistics, drafting the article, and approval of the article; Kurt R. Schumacher and Emily M. Fredericks: Involved in concept/design, data/analysis/interpretation, critical revision of the article, and approval of the article; Kelly E. Rea: Involved in data collection, data analysis/interpretation, statistics, drafting the article, and approval of the article; Sally Eder: Involved in data collection, data analysis/interpretation, statistics, critical revision of the article, and approval of the article; Mary Zamberlan and Jessica Jordan: Collected data, critically revised the article, and approved the article.

\section{ORCID}

Melissa K. Cousino iD http://orcid.org/0000-0002-0041-0830

Emily M. Fredericks (iD http://orcid.org/0000-0002-3110-4983

\section{REFERENCES}

1. Organ Procurement and Transplantation Network. Survival by patient age. 2016. http://optn.transplant.hrsa.gov Accessed April 30, 2017.

2. Uzark K, Griffin L, Rodriguez R, et al. Quality of life in pediatric heart transplant recipients: a comparison with children with and without heart disease. J Heart Lung Transplant. 2012;31:571-578.

3. Foulkes LM, Boggs SR, Fennell RS, Skibinski K. Social support, family variables, and compliance in renal transplant children. Pediatr Nephrol. 1993; 7:185-188.

4. Gerson AC, Furth SL, Neu AM, Fivush BA. Assessing associations between medication adherence and potentially modifiable psychosocial variables in pediatric kidney transplant recipients and their families. Pediatr Transplant. 2004;8:543-550.

5. Soliday E, Kool E, Lande MB. Family environment, child behavior, and medical indicators in children with kidney disease. Child Psychiatry Hum Dev. 2001;31:279-295.

6. DeMaso DR, Kelley SD, Bastardi H, O'Brien P, Blume ED. The longitudinal impact of psychological functioning, medical severity, and family functioning in pediatric heart transplantation. J Heart Lung Transplant. 2004;23:473-480.

7. Wray J, Radley-Smith R. Longitudinal assessment of psychological functioning in children after heart or heart-lung transplantation. $J$ Heart Lung Transplant. 2006;25:345-352.

8. Farley LM, DeMaso DR, D'Angelo E, et al. Parenting stress and parental post-traumatic stress disorder in families after pediatric heart transplantation. J Heart Lung Transplant. 2007;26:120-126.

9. Uzark KC, Crowley DC. Family stress after pediatric heart transplantation. Prog Cardiovasc Nurs. 1989;4:23-27.

10. Uzark KC, Sauer SN, Lawrence KS, Miller J, Addonizio L, Crowley DC. The psychosocial impact of pediatric heart transplantation. J Heart Lung Transplant. 1992;11:1160-1167.

11. Cousino MK, Rea KE, Schumacher KR, Magee JC, Fredericks EM. A systematic review of parent and family functioning in pediatric solid organ transplant populations. Pediatr Transplant. 2017; 21:e12900.

12. Kazak AE, Schneider S, Didonato S, Pai AL. Family psychosocial risk screening guided by the pediatric psychosocial preventative health model (PPPHM) using the Psychosocial Assessment Tool (PAT). Acta Oncol. 2015;54:574-580.

13. Gilleland J, Reed-Knight B, Brand S, et al. Assessment of family psychosocial functioning in survivors of pediatric cancer using the PAT2.0. Psychooncology. 2013;22:2133-2139.

14. Hearps SJ, McCarthy MC, Muscara F, et al. Psychosocial risk in families of infants undergoing surgery for a serious congenital heart disease. Cardiol Young. 2014;24:632-639.

15. Pai AL, Tackett A, Ittenbach RF, Goebel J. Psychosocial Assessment Tool 2.0_General: validity of a psychosocial risk screener in a pediatric kidney transplant sample. Pediatr Transplant. 2012;16: 92-98.

16. Fredericks E, Smith T, Sevecke J, et al. Use of the psychosocial assessment tool to identify risk in families of pediatric liver transplant recipients. Am J Transplant. 2016;16(Suppl. 3):766.

17. Wray J, Radley-Smith R. Depression in pediatric patients before and 1 year after heart or heart-lung transplantation. J Heart Lung Transplant. 2004;23:1103-1110.

18. Kazak AE. Pediatric Psychosocial Preventative Health Model (PPPHM): research, practice, and collaboration in pediatric family systems medicine. Fam Syst Health. 2006;24:381. 
19. Fredericks EM, Zelikovsky N, Aujoulat I, Hames A, Wray J. Post-transplant adjustment-the later years. Pediatr Transplant. 2014;18:675-688.

20. Ikkos ES, Lask B, Whitehead B, Rees P, Graham P. Heart or heartlung transplantation: psychosocial outcome. Pediatr Transplant. 1999;3:301-308.

21. Pai AL, Patiño-Fernández AM, McSherry M, et al. The Psychosocial Assessment Tool (PAT2. 0): psychometric properties of a screener for psychosocial distress in families of children newly diagnosed with cancer. J Pediatr Psychol. 2008;33:50-62.

22. Barrera M, Hancock K, Rokeach A, et al. External validity and reliability of the Psychosocial Assessment Tool (PAT) among Canadian parents of children newly diagnosed with cancer. Pediatr Blood Cancer. 2014;61:165-170.

23. Shemesh E, Lurie S, Stuber ML, et al. A pilot study of posttraumatic stress and nonadherence in pediatric liver transplant recipients. Pediatrics. 2000;105:e29.

24. DeBolt AJ, Stewart SM, Kennard BD, Petrik K, Andrews WS. A survey of psychosocial adaptation in long-term survivors of pediatric liver transplants. Child Health Care. 1995;24:79-96.

25. Newburger PE. Standards for psychosocial care for children with cancer and their families. Pediatr Blood Cancer. 2015;62:S419-S895.

26. Costanzo MR, Dipchand A, Starling $R$, et al. The International Society of Heart and Lung Transplantation Guidelines for the care of heart transplant recipients. J Heart Lung Transplant. 2010;29:914-956.

27. Miloh T, Annunziato R, Arnon R, et al. Improved adherence and outcomes for pediatric liver transplant recipients by using text messaging. Pediatrics. 2009;124:e844-e850.

28. Shemesh E, Annunziato RA, Shneider BL, et al. Improving adherence to medications in pediatric liver transplant recipients. Pediatr Transplant. 2008;12:316-323.

29. Kazak AE, Alderfer M, Rourke MT, Simms S, Streisand R, Grossman JR. Posttraumatic stress disorder (PTSD) and posttraumatic stress symptoms (PTSS) in families of adolescent childhood cancer survivors. J Pediatr Psychol. 2004;29:211-219.

30. Fredericks EM. Transition readiness assessment: the importance of the adolescent perspective. Pediatr Transplant. 2017;21: e12921.

How to cite this article: Cousino MK, Schumacher KR, Rea KE, et al. Psychosocial functioning in pediatric heart transplant recipients and their families. Pediatr Transplant. 2018;22:e13110. https://doi.org/10.1111/petr.13110 